\title{
KSU RADIOCARBON DATES I
}

\author{
OSAMU YAMADA and AKIRA KOBASHIGAWA \\ Faculty of Science, \\ Kyoto Sangyo University, Kyoto 603, Japan \\ INTRODUCTION
}

It was $\mathrm{T}$ Higashimura of Kyoto University who did the first liquid scintillation measurement for ${ }^{14} \mathrm{C}$ in Japan. The External Standard Method (Higashimura et al, 1962) has been accepted the world over as an efficient method of measuring low-level radiation.

Yamada, Higashimura and Sidei (1966) used the methanol synthesis method of ${ }^{14} \mathrm{C}$ dating. Subsequently, a ${ }^{14} \mathrm{C}$ dating laboratory was established at Kyoto Sangyo University by O Yamada in 1969. An Aloka LSC-601 counter for $20 \mathrm{ml}$ vials was introduced in 1971 with an LSC-800 for $100 \mathrm{ml}$ vials in 1973. The appended list includes all samples measured with the I.SC-800 counter from 1975 to 1984 .

\section{METHANOL SYNTHESIS}

Each sample is converted to methanol by the Nystrom formula (Nystrom, Yanko \& Brown, 1948)

$$
\begin{gathered}
4 \mathrm{CO}_{2}+3 \mathrm{LiAl}_{4}=\mathrm{LiAl}\left(\mathrm{OCH}_{3}\right)_{4}+2 \mathrm{LiAlO}{ }_{2} \\
\mathrm{LiAl}\left(\mathrm{OCH}_{3}\right)_{4}+4 \mathrm{ROH}=4 \mathrm{CH}_{3} \mathrm{OH}+\mathrm{LiAl}(\mathrm{OR})_{4}
\end{gathered}
$$

where $\mathrm{R}$ is buthyl carbitol.

Each sample is heated and converted to charcoal in an airtight electric furnace at ca $800^{\circ} \mathrm{C}$ for 2 hours, then boiled in a $1 \%$ solution of $\mathrm{HCl}$ for one hour, washed well in distilled water, and thoroughly dried.

The samples are then placed in a quartz tube and subjected to a stream of heated $\mathrm{N}_{2}$ gas for one hour at $500^{\circ} \mathrm{C}$. $\mathrm{O}_{2}$ is passed through the tube and $\mathrm{CO}_{2}$ gas is made from the charcoal.

The $\mathrm{CO}_{2}$ is then passed through a mixture of $\mathrm{LiAl}_{4}$ and diethyl carbitol for 2 or 3 hours until the reaction ends. Buthyl carbitol is added slowly and abundantly to the mixture and the methanol is separated from the mixture through distillation.

For shell samples, $\mathrm{CO}_{2}$ is derived using diluted $\mathrm{HCl}$ and then transformed into methanol using $\mathrm{LiAlH}_{4}$, as described above. Peat samples are first converted to $\mathrm{CO}_{2}$ and then $\mathrm{CaCO}_{3}$ and then into methanol.

Usually ca $2 \mathrm{~mol}$ of carbon, $2 \mathrm{~mol}$ of $\mathrm{LiAlH}_{4}, 1500 \mathrm{ml}$ of diethyl carbitol, and $1000 \mathrm{ml}$ of buthyl carbitol are used in the process. The approximate yield rate is ca $80 \%$, falling to $60 \%$ after fine distillation. The purity of the final product according to gas chromatography is greater than $99.6 \%$.

\section{MEASUREMENT}

The counting rate and counting efficiency has been measured for all samples to obtain a precise absolute decay rate.

The ${ }^{14} \mathrm{C}$ age is given by the formula

$$
\mathrm{t}=8033 * \ln \left(\mathrm{N}_{0} / \mathrm{N}\right)
$$


where $\mathrm{N}_{0}$ is the concentration of modern ${ }^{14} \mathrm{C}, i e, 95 \%$ of the NBS oxalic acid value and $\mathrm{N}$ is unknown. Anthracite coal was used for the background dead carbon. One standard deviation was used for the error.

When the ${ }^{14} \mathrm{C}$ is measured in a $100 \mathrm{cc}$ teflon vial containing a mixture of $40 \mathrm{~g}$ of methanol, $50 \mathrm{cc}$ of xylene, $0.5 \mathrm{~g}$ of buthyl PBD and $0.05 \mathrm{~g}$ of PBBO, the C-channel counting efficiency was ca $70 \%$ with a background count of $14 \mathrm{cpm}$.

When the recent ${ }^{14} \mathrm{C}$ of $40 \mathrm{~g}$ of methanol is measured over 48 hours, the statistical error of one sigma is ca 20 years including the background error. The oldest age is ca 60,000 BP with $2 \sigma$ criterion measured over a duration of a week.

Isotopic fractionation during the chemical reaction has been extremely small. Fractionation during distillation did occur to some extent but is negligibly smaller than the statistical error for ${ }^{14} \mathrm{C}$ dating by mass spectrography (Shimada \& Yamada, 1977).

\section{ACKNOWLEDGMENTS}

We would like to thank T Higashimura and T Sidei, Kyoto Univ, for guidance in liquid scintillation measurement, and T Hamada, Japan Radioisotope Association, for instruction on $\mathrm{CO}_{2}$ proportional counting method.

\section{GEOLOGIC SAMPIES}

\section{Japan}

\section{Mount Fuji series}

These samples date volcanic history of Mt Fuji. Coll 1960 to 1975 and subm 1975 by H Tsuya, Tokyo Univ and T Ogawa, Japan Volcano Speleo Soc. All lava flows and mud flows are described by Tsuya (1968).

KSU-21. Komakado

$2560 \pm 25$

Wood (chestnut) in mud flow crupted from Mt Fuji, Kisegawa River, Gotenba city, Shizuoka pref ( $\left.35^{\circ} 14^{\prime} 46^{\prime \prime} \mathrm{N}, 138^{\circ} 55^{\prime} 17^{\prime \prime} \mathrm{E}\right)$ alt $345 \mathrm{~m}$.

\section{KSU-22. Nishimarubi}

$2950 \pm 25$

Charcoal in Nim lava flow erupted from young parasitic cinder cone, Fujinomiya city, Shizuoka pref ( $\left.35^{\circ} 22^{\prime} 3^{\prime \prime} \mathrm{N}, 138^{\circ} 40^{\prime} 36^{\prime \prime} \mathrm{E}\right)$ alt $1495 \mathrm{~m}$.

KSU-23. Katsuragawa

$19,190 \pm 150$

Wood (hemlock spruce) in Katsuragawa Older Fuji mud flow, Tsuru city, Yamanashi pref ( $\left.35^{\circ} 33^{\prime} 28^{\prime \prime} \mathrm{N}, 138^{\circ} 54^{\prime} 30^{\prime \prime} \mathrm{E}\right)$ alt $450 \mathrm{~m}$.

KSU-42. Kurozuka

$1460 \pm 25$

Charcoal in ash of Kurozuka parasitic volcano, Susono city, Shizuoka $\operatorname{pref}\left(35^{\circ} 17^{\prime} 18^{\prime \prime} \mathrm{N}, 138^{\circ} 46^{\prime} 55^{\prime \prime} \mathrm{F}\right.$ ). 
KSU-44. Kansuyama

Wood (Japanese cypress) in scoria under Kan lava flow, erupted from young parasitic cinder cone, Susono city (35 $\left.17^{\prime} 17^{\prime \prime} \mathrm{N}, 138^{\circ} 47^{\prime} 3^{\prime \prime} \mathrm{E}\right)$ alt $1230 \mathrm{~m}$

KSU-43. Fudosawa

$1550 \pm 15$

Charcoal under Fud lava flow erupted from small fissure on flank of Mt Fuji, Fuji city, Shizuoka pref $\left(35^{\circ} 19^{\prime} 49^{\prime \prime} \mathrm{N}, 138^{\circ} 44^{\prime} 48^{\prime \prime} \mathrm{E}\right)$ alt $1620 \mathrm{~m}$.

KSU-49. Shibanuta No. 1

$2350 \pm 25$

Wood (Zelkova) in scoria from summit of Mt Fuji, Oyama town, Shizuoka pref $\left(35^{\circ} 21^{\prime} 00^{\prime \prime} \mathrm{N}, 138^{\circ} 53^{\prime} 30^{\prime \prime} \mathrm{E}\right)$ alt $720 \mathrm{~m}$.

KSU-50. Shibanuta No. 2

$1760 \pm 15$

Charcoal, upper of KSU-49.

KSU-57. Takizawa

$1650 \pm 15$

Charcoal in scoria, left bank of Mamabori swamp, Fujiyoshida city, Yamanashi pref $\left(35^{\circ} 25^{\prime} 3^{\prime \prime} \mathrm{N}, 138^{\circ} 46^{\prime} 50^{\prime \prime} \mathrm{E}\right)$ alt $1215 \mathrm{~m}$.

KSU-62. Takamarubi

$1790 \pm 15$

Charcoal in scoria, lower layer of Tam lava flow, Yamanakako village, Yamanashi pref $\left(35^{\circ} 26^{\prime} 57^{\prime \prime} \mathrm{N}, 138^{\circ} 51^{\prime} 42^{\prime \prime} \mathrm{E}\right)$ alt $995 \mathrm{~m}$.

KSU-64. Nanamagari

$1050 \pm 15$

Charcoal in scoria under Fud lava flow, Omote-Fuji hiking road, Fujinomiya city $\left(35^{\circ} 19^{\prime} 50^{\prime \prime} \mathrm{N}, 138^{\circ} 44^{\prime} 5^{\prime \prime} \mathrm{E}\right)$ alt $2000 \mathrm{~m}$.

KSU-73. Karuisaki

$24,330 \pm 110$

Wood (fir) in Older Fuji mud flow, Fujiyoshida city $\left(35^{\circ} 29^{\prime} 48^{\prime \prime} \mathrm{N}\right.$, $\left.138^{\circ} 48^{\prime} 15^{\prime \prime} \mathrm{E}\right)$ alt $450 \mathrm{~m}$.

KSU-74. Jumangoku-Road No. 1

$2570 \pm 15$

Charcoal in scoria under Nim lava flow, Fujinomiya city $\left(35^{\circ} 22^{\prime} 1^{\prime \prime} \mathrm{N}\right.$, $138^{\circ} 40^{\prime} 26^{\prime \prime} \mathrm{E}$ ) alt $1490 \mathrm{~m}$.

KSU-76. Jumangoku-Road No. 2

$2520 \pm 15$

Charcoal in scoria under Yam lava flow, Fujinomiya city $\left(35^{\circ} 22^{\prime} 16^{\prime \prime} \mathrm{N}\right.$, $\left.138^{\circ} 40^{\prime} 23^{\prime \prime} \mathrm{F}\right)$ alt $1495 \mathrm{~m}$.

KSU-79. Jumangoku-Road No. 3

$2700 \pm 20$

Charcoal in scoria under NW 6 lava flow, Fujinomiya city ( $35^{\circ} 21^{\prime} 34^{\prime \prime}$ $\mathrm{N}, 138^{\circ} 40^{\prime} 36^{\prime \prime} \mathrm{E}$ ) alt $1490 \mathrm{~m}$.

KSU-75. Myogadake

$1430 \pm 15$

Charcoal in scoria under SSW 17 lava flow crupted from summit of $\mathrm{Mt}$ Fuji, Fujinomiya city $\left(35^{\circ} 19^{\prime} 10^{\prime \prime} \mathrm{N}, 138^{\circ} 40^{\prime} 26^{\prime \prime} \mathrm{E}\right)$ alt $1530 \mathrm{~m}$. 
KSU-77. Futatsu-tsuka

$310 \pm 15$

Charcoal in scoria erupted from Houei crater of Mt Fuji in AD 1707 , Gotenba city $\left(35^{\circ} 19^{\prime} 45^{\prime \prime} \mathrm{N}, 138^{\circ} 46^{\prime} 50^{\prime \prime} \mathrm{E}\right)$ alt $1804 \mathrm{~m}$.

KSU-78. Inno-Tainai

$1650 \pm 15$

Charcoal from tree mold in Inm lava flow, Gotenba city $\left(35^{\circ} 17^{\prime} 44^{\prime \prime} \mathrm{N}\right.$, $\left.138^{\circ} 51^{\prime} 55^{\prime \prime} \mathrm{E}\right)$ alt $675 \mathrm{~m}$.

KSU-80. Nissawa

$2500 \pm 35$

Charcoal from ancient bonfire, Fujinomiya city $\left(35^{\circ} 18^{\prime} 46^{\prime \prime} \mathrm{N}, 138^{\circ}\right.$ $44^{\prime} 12^{\prime \prime}$ E) alt $1590 \mathrm{~m}$. Comment: suggests human activity.

KSU-81. Taisekiji

$\mathbf{2 4 , 5 2 0} \pm \mathbf{9 0}$

Charcoal in Older Fuji mud flow, Fujinomiya city $\left(35^{\circ} 16^{\prime} 50^{\prime \prime} \mathrm{N}, 138^{\circ}\right.$ $35^{\prime} 15^{\prime \prime} \mathrm{E}$ ) alt $350 \mathrm{~m}$.

\section{KSU-83. Komitake}

$1110 \pm 15$

Charcoal in scoria from Ken 2 lava flow, Narusawa village, Yamanashi pref $\left(35^{\circ} 23^{\prime} 28^{\prime \prime} \mathrm{N}, 138^{\circ} 44^{\prime} 1^{\prime \prime} \mathrm{E}\right)$ alt $2300 \mathrm{~m}$.

\section{KSU-84. Omote-Fuji}

$1150 \pm 20$

Charcoal in scoria under Fud lava flow, hair-pin curve of Omote-Fuji Road, Fujinomiya city $\left(35^{\circ} 19^{\prime} 52^{\prime \prime} \mathrm{N}, 138^{\circ} 44^{\prime} 7^{\prime \prime} \mathrm{E}\right)$ alt $2220 \mathrm{~m}$.

KSU-85. Okuniwa

$1350 \pm 15$

Charcoal under Oniwa 1 lava flow, erupted Oniwa 1 parasitic fissure, Narusawa village $\left(35^{\circ} 23^{\prime} 24^{\prime \prime} \mathrm{N}, 138^{\circ} 41^{\prime} 43^{\prime \prime} \mathrm{E}\right)$ alt $2250 \mathrm{~m}$.

KSU-86. Kenmarubi

$1120 \pm 15$

Charcoal in scoria under Ken 1 lava flow, Fujiyoshida city $\left(35^{\circ} 28^{\prime} 56^{\prime \prime}\right.$ $\mathrm{N}, 138^{\circ} 47^{\prime} 6^{\prime \prime} \mathrm{E}$ ) alt $835 \mathrm{~m}$.

KSU-88. Yamanaka Lake No. 1

$1480 \pm 10$

Wood (larch), mostly outer tree rings, standing at $10 \mathrm{~m}$ depth in lake, Yamanakako village $\left(35^{\circ} 24^{\prime} 50^{\prime \prime} \mathrm{N}, 138^{\circ} 53^{\prime} 0^{\prime \prime} \mathrm{E}\right)$ alt $970 \mathrm{~m}$. Comment: dates fm of Yamanaka Lake dammed by lava flow from Mt Fuji.

KSU-89. Yamanaka Lake No. 2

$1660 \pm 15$

Center tree rings of same sample as KSU-88, with 180 tree rings.

KSU-91. Daifuji golf links

$980 \pm 15$

Charcoal in lava tree mold at Obu lava flow, Fuji city $\left(35^{\circ} 12^{\prime} 15^{\prime \prime} \mathrm{N}\right.$, $138^{\circ} 43^{\prime} 15^{\prime \prime} \mathrm{E}$ ) alt $310 \mathrm{~m}$.

\section{KSU-26. Niijima}

$1130 \pm 20$

Charcoal from lava flow erupted in AD 886, Niijima I. $\left(34^{\circ} 23^{\prime} \mathrm{N}, 139^{\circ}\right.$ $16^{\prime} \mathrm{E}$ ) alt $120 \mathrm{~m}$. Coll and subm by T Sameshima, Shizuoka Univ. 
KSU-60. Kurofuji

$41,900 \pm 860$

Charcoal in loam strata from Kurofuji, Yamanashi pref $\left(35^{\circ} 45^{\prime} \mathrm{N}\right.$, $\left.138^{\circ} 32^{\prime} \mathrm{E}\right)$. Coll and subm by $\mathrm{H}$ Shinohara, Tsuru Coll and T Ogawa. Comment (TO): datum shows same age as Older Fuji, Yatsugatake, Kayagatake and new Hakone, situated in region usually called southern fossa magna.

\section{KSU-72. Fukara}

$1530 \pm 15$

Wood (Cryptomeria) in landslide sand from Hakone volcano, Susono city $\left(35^{\circ} 10^{\prime} 40^{\prime \prime} \mathrm{N}, 138^{\circ} 55^{\prime} 45^{\prime \prime} \mathrm{E}\right)$. Coll and subm by Y Watanabe, Susono city office.

\section{KSU-87. Kobuta-sawa}

$7250 \pm 40$

Wood in landslide sand from Hakone volcano, Tertiary strata, Oshino village, Shizuoka pref $\left(35^{\circ} 24^{\prime} 50^{\prime \prime} \mathrm{N}, 138^{\circ} 53^{\prime} 0^{\prime \prime} \mathrm{E}\right)$ alt $970 \mathrm{~m}$. Coll and subm by $\mathrm{H}$ Tsuya and T Ogawa.

\section{Ohtaki Cave series}

Stalactite from Ohtaki, Gifu pref ( $\left.35^{\circ} 43^{\prime} 27^{\prime \prime} \mathrm{N}, 136^{\circ} 59^{\prime} 44^{\prime \prime} \mathrm{E}\right)$. Coll and subm by H Wada, Shizuoka Univ.

KSU-112. Surface No. 1

$3760 \pm 50$

KSU-114. Surface No. 2

$3370 \pm 40$

KSU-131. Coldest temperature part

$>34,000$

KSU-128. Yogo Lake

$3010 \pm 30$

Tree root from bottom of Yogo Lake, Shiga pref $\left(35^{\circ} 30^{\prime} 40^{\prime \prime} \mathrm{N}, 136^{\circ}\right.$ $11^{\prime} 40^{\prime \prime} \mathrm{E}$ ). Coll and subm by Yogo Educ Bd.

KSU-225. Hyonosen

$3650 \pm 80$

Peat, $90 \mathrm{~cm}$ depth, from Yabu dist, Hyogo pref $\left(35^{\circ} 21^{\prime} \mathrm{N}, 134^{\circ} 1^{\prime} \mathrm{E}\right)$, alt $1470 \mathrm{~m}$. Coll and subm 1978 by M Takeoka, Kyoto Pref Univ.

\section{Amou series}

Samples from Amou marshland, Ono dist, Gifu pref (36 $16^{\prime} \mathrm{N}, 137^{\circ} 1^{\prime}$

E). Coll and subm 1978 by M Yagi, Gifu Univ.

KSU-231. Amou No. 1

$8210 \pm 280$

Peat, $220 \mathrm{~cm}$ depth.

KSU-366. Amou No. 2

$13,320 \pm 190$

Peat, 380 to $400 \mathrm{~cm}$ depth.

\section{KSU-244. Karasuma-Gojo}

$36,290 \pm 800$

Wood, $12.5 \mathrm{~m}$ depth, Kyoto city ( $\left.34^{\circ} 59^{\prime} 36^{\prime \prime} \mathrm{N}, 135^{\circ} 45^{\prime} 44^{\prime \prime} \mathrm{E}\right)$. Coll and subm by $\mathrm{S}$ Ishida, Kyoto Univ. 


\section{KSU-275. Aratozaka}

Wood (chestnut) from bottom of rice field, Obanazawa city, Yamagata pref $\left(38^{\circ} 34^{\prime} \mathrm{N}, 140^{\circ} 30^{\prime} \mathrm{E}\right)$. Coll and subm 1979 by M Takeoka, Kyoto Pref Univ.

\section{KSU-289. Hatchodaira}

Peat, 70 to $80 \mathrm{~cm}$ depth, from Kuta, Kyoto city $\left(35^{\circ} 14^{\prime} \mathrm{N}, 135^{\circ} 50^{\prime} \mathrm{E}\right)$, alt $810 \mathrm{~m}$. Coll and subm 1979 by M Takeoka.

\section{KSU-290. Ukishima}

Peat, 400 to $410 \mathrm{~cm}$ depth, from Shinguu city, Wakayama pref $\left(33^{\circ} 43^{\prime}\right.$ N, $135^{\circ} 59^{\prime} \mathrm{E}$ ). Coll and subm 1979 by M Takeoka.

\section{KSU-291. Sugawara marshland}

Peat, 363 to $373 \mathrm{~cm}$ depth, from Touhaku dist, Tottri pref $\left(35^{\circ} 25^{\prime} \mathrm{N}\right.$, $133^{\circ} 59^{\prime} \mathrm{E}$ ) alt $680 \mathrm{~m}$. Coll and subm 1978 by M Takeoka.

\section{Hananoego series}

Peat from Yaku I., Kumage dist, Kagoshima pref $\left(30^{\circ} 18^{\prime} 40^{\prime \prime} \mathrm{N}, 130^{\circ}\right.$ $30^{\prime} 40^{\prime \prime} \mathrm{E}$ ) alt $1600 \mathrm{~m}$. Coll and subm 1977 by M Takeoka.

KSU-292. Hananoego No. 1

$2450 \pm 80$

Peat, 50 to $60 \mathrm{~cm}$ depth.

KSU-293. Hananoego No. 2

$3280 \pm 100$

Peat, 80 to $90 \mathrm{~cm}$ depth.

\section{Byakushiike series}

Peat from Nishimorogata dist, Miyazaki pref $\left(31^{\circ} 57^{\prime} \mathrm{N}, 130^{\circ} 50^{\prime} \mathrm{E}\right)$ alt $1349 \mathrm{~m}$. Coll and subm 1979 by M Takeoka.

KSU-309. Byakushiike No. 1

$$
\mathbf{0} \pm \mathbf{5 0}
$$

Peat, 150 to $160 \mathrm{~cm}$ depth.

KSU-310. Byakushiike No. 2

Peat, 285 to $295 \mathrm{~cm}$ depth.

\section{KSU-311. Imuta}

Peat, 390 to $400 \mathrm{~cm}$ depth, from Satsuma dist, Kagoshima pref $\left(31^{\circ} 49^{\prime}\right.$ $\mathrm{N}, 130^{\circ} 28^{\prime} \mathrm{E}$ ) alt $295 \mathrm{~m}$. Coll and subm 1979 by M Takeoka.

\section{KSU-314. Okameike}

Peat, 220 to $230 \mathrm{~cm}$ depth, from Uda dist, Nara pref $\left(34^{\circ} 30^{\prime} 54^{\prime \prime} \mathrm{N}\right.$, $136^{\circ} 10^{\prime} 1^{\prime \prime}$ E) alt $710 \mathrm{~m}$. Coll and subm 1979 by M Takeoka.

\section{Joyo series}

Samples from Joyo city, Kyoto pref ( $\left.34^{\circ} 52^{\prime} 6^{\prime \prime} \mathrm{N}, 135^{\circ} 46^{\prime} 0^{\prime \prime} \mathrm{E}\right)$. Coll by J Fukutomi, subm by Educ Bd, Joyo city. 
KSU-340. Joyo No. 1

Wood, 13.5m depth.

KSU-341. Joyo No. 2

Wood, $7 \mathrm{~m}$ depth.

KSU-364. Hirugano

$3790 \pm 100$

Peat, 90 to $100 \mathrm{~cm}$ depth, from Gujo dist, Gifu pref $\left(35^{\circ} 59^{\prime} \mathrm{N}, 136^{\circ} 54^{\prime}\right.$

E). Coll and subm 1980 by M Takeoka.

\section{Hashio series}

Wood from Kouryo, Nara pref ( $\left.34^{\circ} 34^{\prime} 0^{\prime \prime} \mathrm{N}, 135^{\circ} 45^{\prime} 20^{\prime \prime} \mathrm{E}\right)$. Coll and subm by H Okuda.

KSU-431. Hashio No. 1

$3500 \pm 40$

Wood, $4 \mathrm{~m}$ depth, Late Jomon age.

KSU-424. Hashio No. 2

$38,500 \pm 390$

Wood, $5.5 \mathrm{~m}$ depth, upper portion of volcanic tuff layer.

KSU-436. Hashio No. 3

$40,100 \pm 610$

Wood, $6 \mathrm{~m}$ depth, right upper portion of same tuff layer.

\section{KSU-390. Dainaka Lake}

$24,610 \pm 2750$

Wood, $14.8 \mathrm{~m}$ depth, Shiga pref $\left(35^{\circ} 11^{\prime} \mathrm{N}, 136^{\circ} 7^{\prime} \mathrm{E}\right)$. Coll and subm by $\mathrm{S}$ Sasajima, Kyoto Univ, underlying ash of Aira volcano.

KSU-437. Tominaga-Seisakusho

$24,050 \pm 190$

Peat, Nijo-nibo, Kyoto city ( $\left.35^{\circ} 0^{\prime} 37^{\prime \prime} \mathrm{N}, 135^{\circ} 44^{\prime} 2^{\prime \prime} \mathrm{E}\right)$. Coll and subm by $S$ Sasajima.

KSU-438. Seibo Women's College

$19,810 \pm 150$

Peat, Fujinomori, Kyoto city ( $35^{\circ} 57^{\prime} 19^{\prime \prime} \mathrm{N}, 135^{\circ} 46^{\prime} 37^{\prime \prime}$ E). Coll and subm by S Sasajima.

KSU-450. Kigo

$8980 \pm 70$

Peat, 180 to $200 \mathrm{~cm}$ depth, from Tango peninsula, Kyoto pref $\left(35^{\circ} 38^{\prime}\right.$ N, $135^{\circ} 11^{\prime}$ E). Coll and subm 1981 by M Takeoka, Kyoto Pref Univ.

KSU-542. Nawagaike

$480 \pm 180$

Peat, 70 to $80 \mathrm{~cm}$ depth, from Higashitonami dist, Toyama pref $\left(36^{\circ}\right.$ $28^{\prime} 30^{\prime \prime} \mathrm{N}, 136^{\circ} 56^{\prime} 0^{\prime \prime} \mathrm{E}$ ). Coll and subm 1982 by M Takeoka.

KSU-543. Midagahara

$1070 \pm 25$

Peat, 44 to $54 \mathrm{~cm}$ depth, from Tateyama, Nakashinkawa dist, Toyama pref ( $\left.36^{\circ} 34^{\prime} 0^{\prime \prime} \mathrm{N}, 137^{\circ} 33^{\prime} 15^{\prime \prime} \mathrm{E}\right)$. Coll and subm 1982 by M Takeoka. 
KSU-546. Shirakimine

Peat, 68 to $78 \mathrm{~cm}$ depth, from Yao, Nei dist, Toyama pref $\left(36^{\circ} 25^{\prime} 0^{\prime \prime} \mathrm{N}\right.$, $\left.137^{\circ} 7^{\prime} 15^{\prime \prime} \mathrm{E}\right)$. Coll and subm 1982 by M Takeoka.

\section{Mikata Lake series}

Peat from Mikata dist, Fukui pref $\left(35^{\circ} 56^{\prime} \mathrm{N}, 135^{\circ} 54^{\prime} \mathrm{E}\right)$. Coll and subin 1978 by Y Yasuda, Hiroshima Univ.

KSU-640. Mikata No. 1 $2040 \pm 80$

Peat, 190 to $200 \mathrm{~cm}$ depth.

KSU-641. Mikata No. 2 $5670 \pm 100$

Peat, 355 to $375 \mathrm{~cm}$ depth.

KSU-642. Mikata No. 3 $8590 \pm 140$

Peat, 475 to $495 \mathrm{~cm}$ depth.

KSU-465. Mikata No. 4

$15,500 \pm 150$

Peat, 579 to $600 \mathrm{~cm}$ depth.

KSU-467. Mikata No. 5

$18,100 \pm 140$

Peat, 936 to $956 \mathrm{~cm}$ depth.

KSU-650. Mikata No. 6

$20,600 \pm 800$

Peat, 1270 to $1315 \mathrm{~cm}$ depth.

KSU-651. Mikata No. 7

$32,700+6200$

Peat, 2560 to $2584 \mathrm{~cm}$ depth.

Azuchi series

Samples from Azuchi, Gamou dist, Shiga pref. Coll and subm 1982 by Y Tsutsumi, Azuchi town office.

KSU-558. Azuchi No. 1

$2660 \pm 40$

Soil, 56 to $69 \mathrm{~cm}$ depth, from Dainaka $\left(35^{\circ} 9^{\prime} 57^{\prime \prime} \mathrm{N}, 136^{\circ} 7^{\prime} 26^{\prime \prime} \mathrm{E}\right)$.

KSU-567. Azuchi No. 2

$4160 \pm 80$

Soil, 70 to $90 \mathrm{~cm}$ depth, from Dainaka.

KSU-557. Azuchi No. 3

$3210 \pm 40$

Wood from bottom of Dainaka Lake.

KSU-562. Azuchi No. 4

$4200 \pm 110$

Soil from Jionji (35 $\left.8^{\prime} 7^{\prime \prime} \mathrm{N}, 136^{\circ} 7^{\prime} 56^{\prime \prime} \mathrm{E}\right)$.

KSU-563. Azuchi No. 5

$3770 \pm 100$

Soil from Jionji. 
KSU-564. Azuchi No. 6

$1580 \pm 60$ E).

Soil, 14 to $25 \mathrm{~cm}$ depth, from Higashioiso $\left(35^{\circ} 7^{\prime} 40^{\prime \prime} \mathrm{N}, 136^{\circ} 9^{\prime} 50^{\prime \prime}\right.$

KSU-565. Azuchi No. 7

$3350 \pm 70$

Soil, 38 to $46 \mathrm{~cm}$ depth, from Higashioiso.

KSU-566. Azuchi No. 8

$3790 \pm 80$

Soil, 56 to $69 \mathrm{~cm}$ depth, from Higashioiso.

\section{Ichijoji series}

Soil from Kyoto city ( $\left.35^{\circ} 2^{\prime} 22^{\prime \prime} \mathrm{N}, 135^{\circ} 47^{\prime} 43^{\prime \prime} \mathrm{E}\right)$. Coll and subm 1983 by $\mathrm{S}$ Ishida, Kyoto Univ.

KSU-644. Ichijoji No. 1 $8580 \pm 170$

Soil from 2nd black layer.

KSU-645. Ichijoji No. 2

$7790 \pm 100$

Soil from same as No. 1.

KSU-664. Tadachi

$6620 \pm 100$

Peat, 32 to $42 \mathrm{~cm}$ depth, from Minamikiso, Nagano pref $\left(35^{\circ} 39^{\prime} \mathrm{N}\right.$, $137^{\circ} 33^{\prime}$ E). Coll and subm 1983 by M Takeoka, Kyoto Pref Univ.

KSU-665. Kuroauchi

$4520 \pm 80$

Peat, 90 to $100 \mathrm{~cm}$ depth, from Hase, Kamiina dist, Nagano pref $\left(35^{\circ}\right.$ $\left.53^{\prime} \mathrm{N}, 138^{\circ} 10^{\prime} \mathrm{E}\right)$. Coll and subm 1983 by M Takeoka.

\section{KSU-666. Karahanami}

$7420 \pm 70$

Peat, 90 to $100 \mathrm{~cm}$ depth, from Yasaka, Kitaazumi dist, Nagano pref $\left(36^{\circ} 29^{\prime} \mathrm{N}, 137^{\circ} 54^{\prime} \mathrm{E}\right)$. Coll and subm 1983 by M Takeoka.

\section{KSU-647. Kurauchi}

$2430 \pm 30$

Wood (Cryptmeria) from Tango, Takeno dist, Kyoto pref $\left(35^{\circ} 4^{\prime} 37^{\prime \prime} \mathrm{N}\right.$, $135^{\circ} 10^{\prime} 0^{\prime \prime} \mathrm{E}$ ). Coll and subm 1983 by M Takeoka.

\section{KSU-862. Yakumogahara}

$\mathbf{3 3 0 0} \pm \mathbf{5 0}$

Peat, 92 to $102 \mathrm{~cm}$ depth, from Shiga, Shiga dist, Shiga pref $\left(35^{\circ} 37^{\prime} \mathrm{N}\right.$, $\left.135^{\circ} 55^{\prime} \mathrm{E}\right)$. Coll and subm 1984 by M Takeoka.

KSU-863. Fukashimizu

$750 \pm 60$

Peat, 150 to $160 \mathrm{~cm}$ depth, from Imazu, Takashima dist, Shiga pref $\left(35^{\circ} 37^{\prime} \mathrm{N}, 136^{\circ} 0^{\prime} \mathrm{E}\right)$. Coll and subm 1984 by M Takeoka.

\section{KSU-864. Fukakusa}

$$
31,600+2600
$$

Wood from Fukakusa-kuragadani Kyoto city ( $\left.34^{\circ} 57^{\prime} \mathrm{N}, 135^{\circ} 46^{\prime} \mathrm{E}\right)$. Coll 1984 by S Ishidaka and H Okamoto, Kyoto Sci Center for Youth and subm by M Takeoka. 


\section{GG series}

Samples were subm 1983 by H Ohmori, Geog Inst, Tokyo Univ.

KSU-738. GG-1

$$
\mathbf{5 0 , 6 0 0}+\mathbf{8 9 0 0}
$$

Wood, $150 \mathrm{~cm}$ depth, from Kurioka, Oshamanbe, Hokkaido $\left(42^{\circ} 32^{\prime}\right.$ $\left.15^{\prime \prime} \mathrm{N}, 140^{\circ} 21^{\prime} 31^{\prime \prime} \mathrm{E}\right)$. Coll by A Okumura.

KSU-739. GG-2

$>55,100$

Wood, $550 \mathrm{~cm}$ depth, from Horoiwa, Saroma, Hokkaido $\left(44^{\circ} 5^{\prime} 41^{\prime \prime} \mathrm{N}\right.$, $\left.143^{\circ} 53^{\prime} 27^{\prime \prime} \mathrm{E}\right)$. Coll by M Watanabe.

KSU-740. GG-3

$5340 \pm 60$

Coral from Kamikatetsu, Kikai, Kagoshima pref $\left(28^{\circ} 16^{\prime} 30^{\prime \prime} \mathrm{N}, 129^{\circ}\right.$ $\left.56^{\prime} 30^{\prime \prime} \mathrm{E}\right)$. Coll by S Kayane.

\section{KSU-741. GG-4}

$5530 \pm 40$

Coral, same as GG-3.

KSU-742. GG-5

$1610 \pm 90$

Peat, 240 to $250 \mathrm{~cm}$ depth, from Hara, Numazu city, Shizuoka pref $\left(35^{\circ} 8^{\prime} \mathrm{N}, 138^{\circ} 47^{\prime} \mathrm{E}\right)$. Coll by A Matsubara.

KSU-743. GG-6

$7020 \pm 50$

Peat, $500 \mathrm{~cm}$ depth, from Shinden, Maruyama, Chiba pref $\left(35^{\circ} 1^{\prime} 10^{\prime \prime}\right.$ $\left.\mathrm{N}, 139^{\circ} 57^{\prime} 30^{\prime \prime} \mathrm{E}\right)$. Coll by K Kashima.

KSU-744. GG-7

$6980 \pm 460$

Shell, $180 \mathrm{~cm}$ depth, from Amaya, Maruyama, Chiba pref $\left(35^{\circ} 0^{\prime} 40^{\prime \prime} \mathrm{N}\right.$, $139^{\circ} 58^{\prime} 30^{\prime \prime} \mathrm{E}$ ). Coll by K Kashima.

KSU-745. GG-8

$4910 \pm 45$

Coral, same as GG-3.

KSU-746. GG-9

$2540 \pm 60$

Peat, 220 to $225 \mathrm{~cm}$ depth, Higashishihiji, Numazu city, Shizuoka pref $\left(35^{\circ} 7^{\prime} \mathrm{N}, 138^{\circ} 51^{\prime} \mathrm{E}\right)$. Coll by A Matsubara.

\section{HISTORIC SAMPLES}

$$
\text { Japan }
$$

\section{Yamanaka Castle series}

Yamanaka Castle, Mishima city, Shizuoka pref ( $\left.35^{\circ} 9^{\prime} \mathrm{N}, 138^{\circ} 59^{\prime} \mathrm{E}\right)$, was built in 1559, and added on to in 1979. Coll and subm by $\mathrm{H}$ Saitoh, Educ Bd, Mishima city. 
KSU-28. Mumei-Kuruwa F9 No. 1 $\min$.

Wood, sample was $11.89 \mathrm{~g}$ carbon mass; measurement time was 20,200

KSU-104. Mumei-Kuruwa No. 2

$350 \pm 30$

Wood, same sample as KSU-28.

KSU-29. Nishi-Yagura

$320 \pm 5$

Wood from $\mathrm{W} \mathrm{tr}$; $13.36 \mathrm{~g}$ carbon and 34,100 $\mathrm{min}$.

KSU-184. Konrenji

$810 \pm 20$

Wood from Konrenji temple, Kira cho, Aichi pref $\left(34^{\circ} 49^{\prime} \mathrm{N}, 137^{\circ} 6^{\prime}\right.$ E). Coll and subm by $\mathrm{T}$ Kondo, Kyoto Sangyo Univ. Temple was built in early stage of Kamakura Age (AD 1192 to 1332).

\section{Sueki Kama series}

Charcoal, Senboku New Town, Osaka pref (34 $28^{\prime} 34^{\prime \prime}$ N, $135^{\circ} 31^{\prime} 35^{\prime \prime}$ E), from AD 8 th century. Coll and subm by H Nakamura, Ohtani Women's Coll.

KSU-185. TK59 No. 1

$1190 \pm 20$

KSU-189. TK59 No. 2

$1200 \pm 15$

KSU-193. TK59 No. 3

$1220 \pm 30$

\section{Makishima series}

Samples were in Uji River, Kyoto city ( $\left.34^{\circ} 54^{\prime} 11^{\prime \prime} \mathrm{N}, 135^{\circ} 47^{\prime} 38^{\prime \prime} \mathrm{E}\right)$. Coll and subm by Y Murata. Bank of Uji R was constructed by Taiko Hideyoshi in Azuchi-Momoyama Age (AD 1574 to 1602), and occasionally repaired afterwards.

KSU-279. Makishima No. 1

$230 \pm 10$

Wood, stake in Taiko-Bank.

KSU-280. Makishima No. 2

$230 \pm 15$

Wood, another stake in same place as KSU-279.

KSU-281. Makishima No. 3

$\mathbf{3 7 0} \pm 90$

Wood, twig from river sand, $10 \mathrm{~cm}$ depth.

\section{Hizume series}

Samples from iron furnaces from Heian Age (AD 794 to 1191), Shimogamo, Minami-Izu cyo, Shizuoka pref $\left(34^{\circ} 38^{\prime} 10^{\prime \prime} \mathrm{N}, 138^{\circ} 52^{\prime} 0^{\prime \prime} \mathrm{E}\right)$. Coll and subm by $\mathrm{T}$ Satoh.

KSU-307. Hizume No. 1

$920 \pm 25$

Charcoal, B2, middle of Layer 2. 
KSU-308. Hizume No. 2

Charcoal, C3, underlying Layer 3.

\section{ARCHAEOLOGIC SAMPLES}

Japan

\section{Uryudo series}

Uryudo Nishi-iwata site is ancient village of Yayoi Age in Higashiosaka city (34 $39^{\prime} 24^{\prime \prime} \mathrm{N}, 135^{\circ} 36^{\prime} 0^{\prime \prime} \mathrm{E}$ ). Coll and subm 1973 by Y Nakanishi. Comment $(\mathrm{YN})$ : dates cultivation time of waterfield rice in Osaka plain. Results as expected.

\section{KSU-12. Uryudo 12}

$1880 \pm 30$

Wood from UU3PY1, blue-gray layer.

KSU-17. Uryudo 17

$$
2030 \pm 20
$$

Wood from UU3PY15, black sand layer.

KSU-18. Uryudo 18

$$
2460 \pm 30
$$

Wood from UU5CH24, brown clay layer.

\section{KSU-41. Uryudo 20}

Wood from UU3PY15, Pit 2.

KSU-51. Uryudo 19

$2140 \pm 15$

Wood from UU5CH24, brown clay layer.

\section{Toro site series}

Toro, Shizuoka city ( $\left.34^{\circ} 57^{\prime} 3^{\prime \prime} \mathrm{N}, 138^{\circ} 24^{\prime} 33^{\prime \prime} \mathrm{E}\right)$, is typical site of Yayoi Age in Japan. Coll and subm by T Mochizuki, Toro Mus. Comment (TM): expected age: $1800 \mathrm{BP}$.

KSU-31. Toro No. 1

$$
2050 \pm 10
$$

Wood, stake from rice field, $0.5 \mathrm{~km} \mathrm{~S}$ of Toro.

KSU-61. Toro No. 2

Wood, board from same field.

KSU-66. Toro No. 3

Wood, stake from same field.

\section{Shigasato site series}

Shigasato site is W side of Lake Biwa, Shiga pref $\left(35^{\circ} 1^{\prime} \mathrm{N}, 135^{\circ} 52^{\prime} \mathrm{E}\right)$. Coll and subm by S Tanabe. Comment (ST): results of Late and Final Jomon Age as expected, but results of Yayoi Age older. 
KSU-13. Shigasato D

Wood, assoc with first style of Yayoi pottery.

KSU-14. Shigasato A

Wood, same as KSU-13.

KSU-15. Shigasato $\mathrm{C}$

Wood, same as KSU-13.

KSU-16. Shigasato wooden tool

$2940 \pm 10$

Wooden tool, between Late and Final Jomon age.

KSU-40. Shigasato shell

Shell, Final Jomon Age.

\section{Hamane site series}

Samples from salt-making cottages, Hamane, Ohi, Fukui pref $\left(35^{\circ} 32^{\prime}\right.$ $\mathrm{N}, 135^{\circ} 30^{\prime} \mathrm{E}$ ). Coll and subm by M Morikawa, Wakasa Mus. Comment (MM): results seem to be older.

KSU-125. Hamane No. 1

$$
1710 \pm 15
$$

Wood, assoc with Hamane-shiki pottery.

KSU-207. Hamane No. 2

$1860 \pm 40$

Wood, same pottery as KSU-125.

\section{Yotsuike site series}

This site includes many artifacts from Jomon to Kofun Age, Sakai city

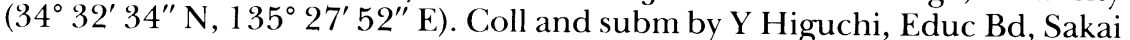
city. Comment $(\mathrm{YH})$ : results as expected except Middle Kofun Age. Results of Middle Kofun Age seem to be older.

KSU-181. Yotsuike No. 1

$1580 \pm 15$

Wood from sand layer in old river, assoc with pot of ca 5 th century.

KSU-183. Yotsuike No. 2

$2120 \pm 15$

Wood from black gray layer at Dist 34, Yayoi Age.

KSU-191. Yotsuike No. 3

$2040 \pm 50$

Wood from gray sand layer at Dist 35, Yayoi Age.

KSU-223. Yotsuike No. 4

$1280 \pm 35$

Charcoal from dark-gray layer in river, Late Kofun Age.

KSU-238. Yotsuike No. 5

$1620 \pm 30$

Wood from black clay layer, Middle Kofun Age. 
KSU-239. Yotsuike No. 6

Wood, Dist 32, Middle Kofun Age.

KSU-240. Yotsuike No. 7

$1580 \pm 40$

Wood from AO gray-brown sand layer, Tr 1 at Dist 32, Middle Kofun Age.

KSU-457. Yotsuike No. 8

$3660 \pm 60$

Charcoal from third phase of Jomon Age at Dist 17, Late Jomon Age.

\section{Ninomiya site series}

Samples from Tsuyama city, Okayama pref $\left(35^{\circ} 2^{\prime} \mathrm{N}, 134^{\circ} 0^{\prime} \mathrm{E}\right)$. Coll and subm by T Takahata, Educ Bd, Okayama pref. Comment (TT): KSU-208 as expected. KSU-182 seems to be older.

\section{KSU-182. Okanotawa}

$1960 \pm 20$

Charcoal, Late Yayoi Age.

KSU-208. Koujinmoto

$930 \pm 25$

Wood, between Late Heian and Kamakura Age.

\section{Torihama site series}

Torihama is important site from Incipient to Late Jomon Age, Mikata-

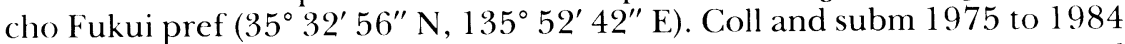
by M Morikawa, Wakasa Mus. Comment (MM): many Jomon ceramics and Oki volcanic ash are dated. Torihama site may be standard of Jomon Age.

KSU-94. TR7501

$5800 \pm 20$

Shell from shell layer, E wall, Sec 1.

KSU-95. TR7502

$5760 \pm 100$

Wood from shell layer, E wall, Sec 1.

KSU-118. TR7503

$5670 \pm 30$

Shell from shell layer, E wall, Sec 1.

KSU-134. TR7504

$5450 \pm 20$

Wood from shell layer, E wall, Sec 1.

KSU-141. TR7505

$5520 \pm 20$

Walnuts from shell layer, E wall, Sec 1.

KSU-154. TR7506

$5810 \pm 25$

Shell from shell layer, E wall Sec 1.

KSU-101. TR7507

$5510 \pm 20$

Wood from Layer 3, S wall, Sec 2. 
KSU-123. TR7508

Wood from Layer 5, S wall, Sec 2.

KSU-102. TR7509

Wood from Layer 6, S wall, Sec 2.

KSU-93. TR7510

Wood from Layer 7, S wall, Sec 2.

KSU-98. TR7511

Wood from Layer 7, S wall, Sec 2.

KSU-92. TR7512

Wood from Layer 10, S wall, Sec 2.

KSU-427. TR80R01

$5130 \pm 100$

Wood, $90 \mathrm{~cm}$ depth, from Layer 14, E wall, Sec 3, with Hajima-kasou 2-shiki pottery.

KSU-405. TR80R02

$5440 \pm 40$

Wood, $135 \mathrm{~cm}$ depth, from Layer 27, E wall, Sec 3, with same pottery as $\mathrm{KSU}-427$.

KSU-399. TR80R03

$5500 \pm 45$

Wood, $140 \mathrm{~cm}$ depth, from Layer 27 , E wall, Sec 3, with same pottery as KSU-427.

KSU-361. TR80R04

$9780 \pm 60$ tery.

Wood, $160 \mathrm{~cm}$ depth, from Layer 31, Sec 2 to 3, with Tajomon pot-

KSU-397. TR80R05

$10,080 \pm 60$

Wood, $170 \mathrm{~cm}$ depth, from Layer 31, E wall, Sec 3, with same pottery as $\mathrm{KSU}-361$.

KSU-404. TR80R06

$10,320 \pm 60$ 361 .

Wood, $190 \mathrm{~cm}$ depth, from Layer 33, Sec 3, with same pottery as KSU-

KSU-419. TR80L01

$4790 \pm 25$

Wood, $60 \mathrm{~cm}$ depth, Layer 5, Sec 3.

KSU-396. TR80L02

$5780 \pm 20$ ash

Wood, $70 \mathrm{~cm}$ depth, from Layer 7, $5 \mathrm{H}$, Sec 2, upper Akahoya volcanic 
KSU-395. TR80L03

$7010 \pm 30$

Charcoal, $80 \mathrm{~cm}$ depth, from Layer 8, 5I, Sec 2, under Akahoya volcanic ash.

KSU-409. TR80L04

$8130 \pm 30$

Wood, $185 \mathrm{~cm}$ depth, from Layer 22 to $23,15 \mathrm{C}$, Sec 3, with Oshigatamon pottery.

KSU-389. TR80L05

$8190 \pm 300$

Wood, $205 \mathrm{~cm}$ depth, from Layer 24 , Sec 4 , upper Oki volcanic ash, with same pottery as KSU-409.

KSU-382. TR80L06

$8970 \pm 120$

Soil, $208 \mathrm{~cm}$ depth, from bottom of Oki volcanic ash, with same pottery as KSU-409.

KSU-388. TR80L07

$\mathbf{9 1 7 0} \pm \mathbf{5 0}$

Wood, $210 \mathrm{~cm}$ depth, from Layer 26 , under Oki volcanic ash, Sec 4, with same pottery as KSU-409.

KSU-485. TR80L08

$11,850 \pm 100$

Wood, $280 \mathrm{~cm}$ depth, from Layer 32 , Tr E, Sec 3 .

KSU-478. TR80L09

$11,900 \pm 110$

Wood, $280 \mathrm{~cm}$ depth, from Layer 32 , Tr E, Sec 3.

KSU-400. TR80L10

$11,470 \pm 70$

Wood, $305 \mathrm{~cm}$ depth, from Layer 37, Sec 3.

KSU-484. TR80L11

$11,500 \pm 100$

Wood, $305 \mathrm{~cm}$ depth, from Layer 37, Sec 3.

KSU-471. TR80L12

$12,100 \pm 100$

Wood, $305 \mathrm{~cm}$ depth, from Layer 37, Sec 3.

KSU-477. TR80L13

$12,100 \pm 130$

Wood, $305 \mathrm{~cm}$ depth, from Layer 37, Sec 3 .

KSU-571. TR8201

$3780 \pm 50$

Wood, piece of canoe, from Layer 3, Sec 2 to 3.

KSU-572. TR8202

$3680 \pm 35$

Wood, stick from canoe, from Layer 3, Sec 2.

KSU-1012. TR8301

$5220 \pm 35$

Wood, $95 \mathrm{~cm}$ depth, from Layer 13, 23B $21 \mathrm{D}$, Sec 1, with Kitashirakawa-kasou 2-c-shiki pottery. 
KSU-1019. TR8401

$5170 \pm 30$

Wood, $80 \mathrm{~cm}$ depth, from Layer 37 , Sec 2, with Kitashirakawa-kasou 2-b-shiki pottery.

KSU-1013. TR8302

$5330 \pm 30$

Wood, $170 \mathrm{~cm}$ depth, from Layer 31 to $34,22 \mathrm{E}$, Sec 1 , with same pottery as KSU-1019.

KSU-1014. TR8303

$\mathbf{5 9 1 0} \pm \mathbf{3 0}$

Wood, $95 \mathrm{~cm}$ depth, from under Layer 73, 33B, Sec 3, with Hajimakasou 2-shiki pottery.

KSU-1020. TR8402

$5500 \pm 40$

Wood, $110 \mathrm{~cm}$ depth, from Layer $39,23 \mathrm{H}$ 25I $25 \mathrm{~K}$, Sec 2 , with same pottery as KSU-1014.

KSU-1021. TR8403

$5200 \pm 40$

Wood, $120 \mathrm{~cm}$ depth, from Layer $41,21 \mathrm{~L}$, Sec 2 , with same pottery as KSU-1014.

KSU-1022. TR8404

$7250 \pm 60$ tery.

Peat, $30 \mathrm{~cm}$ depth, from Layer 49a, 29M, Sec 2, with Initial Jomon pot-

KSU-1023. TR8405

$8330 \pm 45$ pottery.

Peat, $40 \mathrm{~cm}$ depth, from Layer 49b, 29M, Sec 2, with Oshigata-mon

KSU-1024. TR8406

$9120 \pm 80$

Peat, $60 \mathrm{~cm}$ depth, from Layer $51,29 \mathrm{M}$, Sec 2 , with same pottery as KSU-1023.

KSU-1015. TR8304

$10,070 \pm 60$ tery.

Wood, $130 \mathrm{~cm}$ depth, from Layer $80,39 \mathrm{~B}$, Sec 3, with Tajomon pot-

KSU-1016. TR8305

$10,070 \pm 45$

Wood, $150 \mathrm{~cm}$ depth, from Layer $82,38 \mathrm{D} 39 \mathrm{~B} 37 \mathrm{~B}$, Sec 3 , with same pottery as KSU-1015.

KSU-1025. TR8407

$10,270 \pm 45$

Wood, $105 \mathrm{~cm}$ depth, from Layer $60,29 \mathrm{M}$, Sec 2, with same pottery as KSU-1015.

KSU-1026. TR8408

$10,130 \pm 45$

Peat, $105 \mathrm{~cm}$ depth, from Layer $60,29 \mathrm{M}$, Sec 2 , with same pottery as KSU-1015. 
KSU-1017. TR8306

$10,290 \pm 45$

Wood, $180 \mathrm{~cm}$ depth, from Layer $85,36 \mathrm{E} 38 \mathrm{C} 34 \mathrm{D}$, Sec 3 , with Tsumegata-mon pottery.

KSU-1027. TR8409

$10,770 \pm 160$

Wood, $165 \mathrm{~cm}$ depth, from Layer $62,22 \mathrm{M}$, Sec 2 , with same pottery as KSU-1017.

KSU-1028. TR8410

$11,830 \pm 60$

Wood, $185 \mathrm{~cm}$ depth, from Layer 66, 29M, Sec 2, with Ryusenmon pottery.

KSU-1029. TR8411

$11,800 \pm 60$

Wood, $180 \mathrm{~cm}$ depth, from Layer 66 , Sec 3 , with same pottery as KSU1028 .

KSU-1030. TR8412

$11,700 \pm 60$

Wood, 200cm depth, from Layer 67, 22M, Sec 2.

KSU-1018. TR8307

$11,730 \pm 50$

Wood, $270 \mathrm{~cm}$ depth, from Layer 90, Tr A, Sec 2.

KSU-1031. TR8413

$11,870 \pm 50$

Wood, $225 \mathrm{~cm}$ depth, from Layer 68, 22M, Sec 2.

\section{Yoshidaminami site series}

Samples from Tarumi-ku, Kobe city ( $\left.34^{\circ} 40^{\prime} \mathrm{N}, 134^{\circ} 49^{\prime} \mathrm{E}\right)$. Coll and subm by $\mathrm{S}$ Tanabe, Nara Univ. Comment (ST): expected age: Kofun to Nara Age. Results of Nara Age as expected, but results of Kofun Age older.

KSU-194. Yoshidaminami No. 1

$1890 \pm 30$

Wooden stake, 3YM, Kofun Age.

KSU-229. Yoshidaminami No. 2

$1760 \pm 50$

Wood, 3YM-SB26.

KSU-235. Yoshidaminami No. 3

$1380 \pm 70$

Wood, SE1.

KSU-227. Yoshidaminami No. 4

$1290 \pm 40$

Wood, 1YMSE2, Nara Age.

KSU-220. Yoshidaminami No. 5

$1260 \pm 70$

Wood, 2YM. 
Shell from Tahara, Aichi pref $\left(34^{\circ} 40^{\prime} 41^{\prime \prime} \mathrm{N}, 137^{\circ} 17^{\prime} 4^{\prime \prime} \mathrm{E}\right)$. Coll and subm by $S$ Sumida, Nagoya Univ. Comment (SS): assoc with Final Jomon pottery. Result as expected.

\section{KSU-215. Hassaki site}

$$
6880 \pm 35
$$

Shell from Ohbu city, Aichi pref $\left(35^{\circ} 0^{\prime} 39^{\prime \prime} \mathrm{N}, 137^{\circ} 0^{\prime} 14^{\prime \prime} \mathrm{E}\right)$. Coll and subm by S Sumida. Comment (SS): assoc with Early Jomon pottery. Result as expected.

\section{KSU-216. Asahi site}

$$
2600 \pm 40
$$

Shell from Nagoya city $\left(35^{\circ} 12^{\prime} 50^{\prime \prime} \mathrm{N}, 136^{\circ} 51^{\prime} 10^{\prime \prime} \mathrm{E}\right)$. Coll and subm by S Sumida. Comment (SS): assoc with Early Yayoi pottery. Result seems to be much older.

\section{Tarumiminami site series}

Samples from Suita city, Osaka pref ( $\left.34^{\circ} 45^{\prime} 37^{\prime \prime} \mathrm{N}, 135^{\circ} 30^{\prime} 16^{\prime \prime} \mathrm{E}\right)$. Coll and subm by M Fujiwara, Educ Bd, Suita city. Comment (MF): assoc with Kofun pottery.

KSU-268. Tarumiminami No. 1 $1750 \pm 10$

Wood.

KSU-269. Tarumiminami No. 2 $1750 \pm 15$

Wood.

KSU-540. Tarumiminami No. 3

$1780 \pm 20$

Wood.

\section{Senpukuji site series}

Samples from Sasebo city, Nagasaki pref ( $\left.33^{\circ} 11^{\prime} 54^{\prime \prime} \mathrm{N}, 129^{\circ} 44^{\prime} 5^{\prime \prime} \mathrm{E}\right)$. Coll and subm by M Asou, Chiba Univ. Comment (MA): assoc with microlith and Jokon-mon pottery. Expected age: 8000-10,000 BP.

KSU-276. Senpukuji No. 1

$10,300 \pm 200$

Soil from Layer $7 \mathrm{~b}$.

KSU-277. Senpukuji No. 2

$$
10,160 \pm 150
$$

Soil from Layer 8.

\section{Kyodai site series}

Samples from Kyoto city $\left(35^{\circ} 1^{\prime} 44^{\prime \prime} \mathrm{N}, 135^{\circ} 47^{\prime} 35^{\prime \prime} \mathrm{E}\right)$. Coll and subm by T Izumi, Kyoto Univ. Comment (TI): expected period: Final Jomon Age.

KSU-304. Kyodai No. 1

$2000 \pm 10$

Wood from Layer 2. 
KSU-286. Kyodai No. 2

Soil from Layer 2.

KSU-299. Kyodai No. 3

Wood from blue-gray layer.

KSU-283. Kyodai No. 4

Wood from blue-gray layer.

KSU-287. Kyodai No. 5

Soil from Layer 4.

KSU-282. Kyodai No. 6

Wood from Layer 4.

KSU-284. Kyodai No. 7

Wood from Layer 4.

KSU-288. Kyodai No. 8

Soil from Layer 4.

KSU-334. Teradani site

Charcoal from Late Stone Age site, Iwata city, Shizuoka pref $\left(34^{\circ} 46^{\prime}\right.$ $\mathrm{N}, 137^{\circ} 51^{\prime} \mathrm{E}$ ). Coll and subm by T Suzuki, Heian Mus. Comment (TS): assoc with backed blade. Result as expected.

\section{Hegi Cave series}

Samples from Honyamakei, Ohita pref ( $\left.34^{\circ} 29^{\prime} 20^{\prime \prime} \mathrm{N}, 131^{\circ} 12^{\prime} 24^{\prime \prime} \mathrm{E}\right)$. Coll and subm 1979 to 1983 by M Kagawa, Beppu Univ. Comment (MK): assoc with many human bones from Initial to Late Jomon Age. Dates of shell in river ca 1200 yr older than plants. Results as expected.

\section{KSU-337. Hegi No. 1}

$6510 \pm 45$

Shell from Layer $4 \mathrm{a}$.

KSU-346. Hegi No. 2

$7310 \pm 20$

Shell from Layer $4 b$.

KSU-347. Hegi No. 3

$5150 \pm 40$

Soil from Layer $4 \mathrm{a}$.

KSU-353. Hegi No. 4

$6400 \pm 50$

Soil from Layer $4 b$.

KSU.354. Hegi No. 5

$7590 \pm 50$

Shell from Layer $5 b$. 
KSU-384. Hegi No. 6

$5850 \pm 60$

Soil from Layer 5.

KSU-385. Hegi No. 7

$7510 \pm 50$

Soil from Layer 5 .

KSU-411. Hegi No. 8

$6470 \pm 45$

Soil from Layer $4 \mathrm{~b}$.

KSU-412. Hegi No. 9

$3640 \pm 40$

Soil from Layer $3 \mathrm{a}$.

KSU-638. Hegi No. 10

$10,700 \pm 900$

Charcoal from Layer $5 \mathrm{a}$.

KSU-639. Hegi No. 11

$11,100 \pm 800$

Charcoal from Layer $7 \mathrm{c}$.

\section{Hiruzen site series}

Samples from Yatsuka, Maniwa dist, Okayama pref $\left(35^{\circ} 18^{\prime} \mathrm{N}, 133^{\circ} 42^{\prime}\right.$ E). Coll and subm 1983 by Y Kamaki, Okayama Coll Sci. Comment (YK): assoc with backed blade. Results as expected.

KSU-568. Hiruzen No. 1

$18,400 \pm 230$

Peat from Layer 6, upper Odori volcanic ash.

KSU-550. Hiruzen No. 2

$24,000+4000$

$-3000$

Charcoal from Layer 10, underlying AT volcanic ash.

KSU-612. Hiruzen No. 3

$23,400 \pm 500$

Same sample as No. 2.

\section{Hironokita site series}

Charcoal from Toyoda, Shizuoka pref $\left(34^{\circ} 44^{\prime} \mathrm{N}, 137^{\circ} 50^{\prime} \mathrm{E}\right)$. Coll and subm 1983 by H Yamashita, Heian Mus. Comment (HY): assoc with backed blade, point and microblade. Results as expected.

KSU-671. Hironokita No. 1

$22,300 \pm 800$

Charcoal from right upper AT volcanic ash.

KSU-672. Hironokita No. 2

$22,100 \pm 800$

Charcoal from upper AT volcanic ash.

KSU-673. Hironokita No. 3

$25,300+2000$

Charcoal underlying AT volcanic ash. 


\section{Kannami site series}

Samples coll from rice field of Kannami-cho, Shizuoka pref $\left(35^{\circ} 5^{\prime} \mathrm{N}\right.$, $\left.138^{\circ} 57^{\prime} \mathrm{E}\right)$. Coll and subm by Y Nagano. Comment (YN): results as expected.

KSU-355. Kannami No. 1

$1870 \pm 15$

Wooden stake from A8 grid, Late Yayoi Age.

KSU-356. Kannami No. 2

$1750 \pm 20$

Wood from Z7 grid, between Late Yayoi and Early Kofun Age.

KSU-359. Kannami No. 3

$1620 \pm 15$

Wood from Z4 grid, Kofun Age.

KSU-362. Kannami No. 4

$1850 \pm 20$

Wood from Z4 grid, W-29 Layer 5, Late Yayoi Age.

KSU-414. Kurosaki site

$3310 \pm 30$

Shell from Kitakyusyu city, Fukuoka pref $\left(33^{\circ} 51^{\prime} 31^{\prime \prime} \mathrm{N}, 130^{\circ} 45^{\prime} 55^{\prime \prime}\right.$ E). Coll and subm by M Tachibana, Beppu Univ. Comment (MT): assoc with Late Jomon pottery. Result as expected.

KSU-415. Kanegasaki site

$3480 \pm 25$

Shell from Genkai, Fukuoka pref ( $\left.33^{\circ} 52^{\prime} 10^{\prime \prime} \mathrm{N}, 130^{\circ} 31^{\prime} 57^{\prime \prime} \mathrm{E}\right)$. Coll and subm by M Tachibana. Comment (MT): pottery type is older than KSU414. Result as expected.

\section{Hyakkengawa site series}

Wood from Kanemoto, Okayama pref $\left(34^{\circ} 40^{\prime} \mathrm{N}, 133^{\circ} 57^{\prime} \mathrm{E}\right)$. Coll and subm by T Takahata, Educ Bd, Okayama pref. Comment (TT): results seem to be older.

KSU-426. Hyakkengawa No. 1

$2180 \pm 15$

Wood, Yayoi Age.

KSU-429. Hyakkengawa No. 2

$1900 \pm 20$

Wood, Early Kofun Age.

\section{Bibi site series}

Samples coll 1980 by $\mathrm{N}$ Kimura, subm 1980 by R Asai, Center Archaeol Research Hokkaido, Chitose city, Hokkaido $\left(42^{\circ} 46^{\prime} \mathrm{N}, 141^{\circ} 39^{\prime}\right.$ E). Comment (NK): results as expected except for KSU-374.

KSU-367. Bibi No. 1

$3970 \pm 35$

Charcoal, Middle Jomon Age.

KSU-370. Bibi No. 2

$25,320 \pm 1010$

Charcoal underlying Yop3 volcanic ash, Stone Age. 
KSU-372. Bibi No. 3

$14,410 \pm 2090$

Charcoal from upper Shikotsu volcanic ash, Stone Age.

KSU-374. Bibi No. 4

$5450 \pm 450$

Charcoal, $0.315 \mathrm{~g}$ carbon, Late Jomon Age.

\section{Misawa site series}

Samples coll 1980 by N Kimura, subm 1980 by R Asai, from Tomakomai, Hokkaido ( $\left.42^{\circ} 45^{\prime} \mathrm{N}, 141^{\circ} 39^{\prime} \mathrm{E}\right)$. Comment (NK): results as expected.

KSU-358. Misawa No. 1

$5620 \pm 25$

Shell, Early Jomon Age.

KSU-360. Misawa No. 2

$5480 \pm 35$

Shell, Early Jomon Age.

KSU-375. Misawa No. 3

$3510 \pm 100$

Wood, Late Jomon Age.

KSU-365. Suehiro site

$1140 \pm 25$

Charcoal from Chitose city, Hokkaido $\left(42^{\circ} 50^{\prime} \mathrm{N}, 141^{\circ} 39^{\prime} \mathrm{E}\right)$, with Satsumon pottery. Coll and subm 1980 by T Ohtani Educ Bd, Chitose city. Comment (TO): result as expected.

\section{Shadai site series}

Samples from Shiraoi, Hokkaido $\left(42^{\circ} 32^{\prime} \mathrm{N}, 141^{\circ} 26^{\prime} \mathrm{E}\right)$. Coll 1980 by Y Taneichi, subm 1980 by R Asai. Comment (YT): results as expected.

KSU-368. Shadai No. 1

$2910 \pm 45$

Charcoal, Final Jomon Age.

KSU-369. Shadai No. 2

$190 \pm 45$

Driftwood.

\section{Kawakami B site series}

Samples from Noboribetsu city, Hokkaido $\left(42^{\circ} 24^{\prime} \mathrm{N}, 141^{\circ} 11^{\prime} \mathrm{E}\right)$. Subm 1980 and 1982 by R Asai. Comment (YN): expected age: 3000-7000 BP.

KSU-376. Kawakami No. 1

$5170 \pm 90$

Charcoal, J-1 7-a, inside Jomon pottery. Coll 1980 by Y Nakamura.

KSU-584. Kawakami No. 2

$3250 \pm 80$

Charcoal, L-93-376. Coll 1982 by H Hata.

KSU-585. Kawakami No. 3

$3200 \pm 120$

Charcoal, J-92-d-570. Coll by H Hata. 
KSU-586. Kawakami No. 4

Charcoal, L-93-a-152. Coll by H Hata.

\section{Chitose site series}

Samples from Noboribetsu city, Hokkaido $\left(42^{\circ} 24^{\prime} \mathrm{N}, 141^{\circ} 11^{\prime} \mathrm{E}\right)$. Subm 1980 and 1982 by $\mathrm{R}$ Asai. Comment (YN and $\mathrm{AO}$ ): results as expected.

KSU-377. Chitose 4 Site No. 1

$$
4060 \pm 110
$$

Charcoal, K-16-a, Jomon Age. Coll 1980 by Y Nakamura.

KSU-378. Chitose 4 Site No. 2 $3600 \pm 80$

Charcoal, J-16-b. Coll by Y Nakamura.

KSU-580. Chitose 5 Site No. 1

$3900 \pm 120$

Charcoal, H-3, Layer 3, between Middle and Late Jomon Age. Coll 1982 by A Oniyanagi.

KSU-581. Chitose 5 Site No. 2

$3920 \pm 180$

Charcoal, H-6. Coll by A Oniyanagi.

KSU-582. Chitose 5 Site No. 3

$3170 \pm 260$

Charcoal, H-13. Coll by A Oniyanagi.

\section{Kojohama site series}

Samples from Shiraoi, Hokkaido $\left(42^{\circ} 28^{\prime} \mathrm{N}, 141^{\circ} 9^{\prime} \mathrm{E}\right)$. Subm 1980 and 1982 by R Asai.

\section{KSU-379. Kojohama 4 site}

$3860 \pm 40$

Charcoal, Middle Jomon Age. Coll 1980 by Y Taneichi. Comment (YT): expected age: $4500-5000 \mathrm{BP}$.

KSU-380. Kojohama 3 Site No. 1 $3820 \pm 60$

Charcoal. Coll by Y Taneichi. Comment (YT): expected age: $6000 \mathrm{BP}$.

KSU-559. Kojohama 3 Site No. 2 $3740 \pm 40$

Charcoal. Coll 1982 by K Satoh. Comment (KS): expected period: Middle Jomon Age. Result as expected.

KSU-583. Kojohama 3 Site No. 3 $7450 \pm 400$

Charcoal. Coll by K Satoh. Comment (KS): assoc with Initial Jomon pottery. Result as expected.

\section{Kabukai site series}

Samples from Rebun I., Hokkaido $\left(45^{\circ} 24^{\prime} \mathrm{N}, 141^{\circ} 0^{\prime} \mathrm{E}\right)$, with Satsumon pottery. Coll and subm 1977 by H Ohi, Hokkaido Univ. 
KSU-192. RKA 1

Charcoal. Comment (HO): younger than KSU-209.

KSU-209. RKA 2

$1270 \pm 30$

Charcoal. Comment (HO): assoc with Satsumon 2-3 pottery. Expected age: $1200 \mathrm{BP}$.

KSU-210. RKA 3

$1400 \pm 25$

Charcoal. Comment (HO): assoc with same pottery as KSU-209.

KSU-211. RKA 4

$2040 \pm 60$

Charcoal. Comment (HO): expected age is older than KSU-209.

\section{Komaba 7 site series}

Samples from Shizunai, Hokkaido $\left(42^{\circ} 21^{\prime} \mathrm{N}, 142^{\circ} 21^{\prime} 30^{\prime \prime} \mathrm{E}\right)$. Coll 1980 and subm 1981 by T Kohara, Educ Bd, Shizunai-cho. Comment (TK): results as expected.

KSU-463. SP-No. 1

$7370 \pm 200$

Charcoal, PH-7, with Akatsuki-shiki pottery.

KSU-454. SP-No. 2

$7310 \pm 140$

Charcoal, PH-15.

KSU-464. SP-No. 3

$8840 \pm 200$

Charcoal, $\mathrm{PH}-18$.

KSU-472. SP-No. 4

$8730 \pm 90$

Charcoal, PH-20.

KSU-462. SP-No. 5

$8730 \pm 130$

Charcoal, PH-20.

\section{REFERENCES}

Higashimura, T, Yamada, O, Nohara, N and Sidei, T, 1962, External standard method for determination of efficiency of liquid scintillation counting: Internatl Jour Appl Radiation \& Isotopes, v 13, p 308-309.

Nystrom, R F, Yanko, W and Brown, W G, 1948, Reduction of carbon dioxide to methanol by lithium aluminum hydride: Am Chem Jour, v 70, p 441.

Shimada, H and Yamada, O, 1977, The isotopic effect in methanol synthesis for the liquid scintillation ${ }^{14} \mathrm{C}$ dating: Acta Humanistica Sci Univ Sangio Kyotiensis, v 6, no. 3, p 7889.

Sidei, T, Higashimura, T and Yamada, O, 1962, The concentration of carbon-14 in ethanol: Jour Radiation Research, v 3, no. 3, p 153-157.

Tsuya, H, 1968, Geologic map of Mt Fuji: Geol Survey of Japan.

Yamada, O, Higashimura, T and Sidei, T, $1966,{ }^{14} \mathrm{C}$-dating by liquid scintillation counting: Radioisotopes, $v 15$, no. 2, p 73-76. 